\title{
INTERVENCIÓN ARQUEOLÓGICA EN LA VILLA ROMANA “EL TESORILLO” DEL CORTIJO DE TIENA \\ LA ALTA (MOCLÍN. GRANADA). PROCESO DE RESTAURACIÓN Y ARRANQUE DE UN MOSAICO
}

\author{
Manuel Angel CASTILLO RUEDA (*) \\ Margarita ORFILA PONS (**) \\ María Isabel MANCILLA CABELLO (*) \\ Luis Alberto CARRETERO LÓPEZ (***) \\ Miguel DÍAZ MARÍN (***) \\ Pilar ARAGÓN MAZA (***)
}

(*) Grupo de Investigación de Arqueología en Época Clásica y Antigüedad Tardía en Andalucia Oriental. Universidad de Granada.

(**) Universidad de Granada.

(***) Restauradores.

\section{Introducción}

En el presente estudio se recogen los resultados obtenidos tras el proceso de excavación arqueológica, restauración y extracción acometido con carácter de urgencia en el mosaico y restos arqueológicos de época romana hallados en las inmediaciones del Cortijo de Tiena La Alta (Moclín, Granada), concretamente en el pago conocido como "El Tesorillo". Dichos vestigios pertenecen a la pars urbana de una villa hispanorromana. La intervención ha sido aprobada y subvencionada por la Consejería de Cultura de la Dirección General de Bienes Culturales de la Junta de Andalucía en el año 1997.

Esta excavación se ha realizado entre los días 14 de octubre y 15 de diciembre de 1997, concluyéndose la extracción del mosaico puesto al descubierto el día 22 de enero. Los trabajos se han llevado a cabo por parte de un equipo de investigadores formado por el director, Manuel Ángel Castillo Rueda, María Isabel Mancilla Cabello, además de la colaboración de la Dra. Margarita Orfila Pons y tres restauradores, Luis Alberto Carretero López, Miguel Díaz Marín y Pilar Aragón Maza ${ }^{1}$

\footnotetext{
' Desde estas líneas queremos expresar nuestro más sincero agradecimiento por el apoyo prestado a todos aquellos que han hecho posible la realización de esta intervención, sobretodo a Dña. Carmela Pérez de la Delegación de Cultura de Granada, a D. Rafael Gómez y Dĩa. Concepción San Martín, restaurador y directora respectiva-
} 


\section{Situación del yacimiento. Consideraciones previas}

El yacimiento arqueológico de la villa romana del Cortijo de Tiena la Alta está ubicado sobre una loma o cerro amesetado, que se prolonga desde las elevaciones conocidas como el Monte de Tiena la Alta, junto al Cortijo que lleva el mismo nombre, en el pago de "El Tesorillo"2. Dicho topónimo es tan significativo como la propia realidad a la que se refiere. Se accede a través de un camino que se desvía de la carretera que enlaza la pedanía de Tiena con la carretera nacional 432 Granada-Cór-

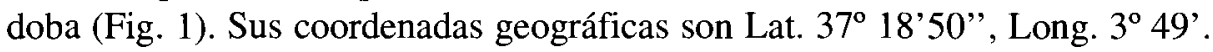

En el terreno donde se halla enclavado el asentamiento destaca la gran cantidad de restos materiales de época romana que aparecen en superficie, abarcando un área de unos $15.000 \mathrm{~m}^{2}$. de dispersión (véase Fig. 2 espacio señalado como GR-MC-11) destacando las abundantes teselas en blanco y negro, los bloques de sillarejo y los fragmentos de estuco pintado, así como una gran diversidad de fragmentos de cerámica hallados en superficie que permitieron datar, grosso modo, la ocupación de este asentamiento rural romano desde el s. I al V d.C

Próximo a este primer espacio, se apreció otra zona (véase Fig. 2 el espacio señalado como GR-MC-12) cubierta por materiales arqueológicos que, dado su carácter (básicamente tégulas), se podría pensar que se trata de la necrópolis adscrita a la villa.

En cuanto a la situación en la que se encontraban los restos antes de la intervención hay que mencionar que la propiedad está plantada de olivos. Por esta circunstancia se han venido llevando a cabo actuaciones puntuales y periódicas sobre el terreno que han afectado en mayor o menor medida al registro arqueológico de este yacimiento (arado, abonado, etc.). Pero el problema que más ha dañado al registro arqueológico ha sido la realización de fosas con ayuda de una máquina retro-excavadora para plantar olivos hace una década. Estas fosas han alterado de forma considerable los restos con los que han topado, como más adelante se detallará. Junto a este

mente, del Museo Arqueológico y Etnológico Provincial de Granada y al Excmo. Ayuntamiento de Moclín por todas las facilidades que nos ha brindado desde el primer día, poniendo el Sr. alcalde a nuestra disposición su apoyo técnico y humano, así como a la Escuela Taller de Moclín. También queremos hacerlo extensivo a D. Manuel López funcionario del Ayuntamiento de Moclín y al propietario de la finca.

${ }^{2}$ Durante las prospecciones arqueológicas llevadas a cabo en el término de Íllora, enmarcadas dentro del Proyecto de Estudio del Poblamiento de la Vega de Granada durante la Prehistoria Reciente, Época Clásica y Antigüedad Tardía, el equipo que dirigía esta actividad fue alertado de la existencia de este yacimiento.

${ }^{3}$ Esta nomenclatura que siguió dicho proyecto se ha tenido presente también durante la excavación, y en la que se hace referencia al número (11) que ocupa dentro de los yacimientos existentes en el término de Moclín (MC). 
punto, el otro problema acuciante con el que se ha tenido que enfrentar el propio yacimiento han sido los grandes hoyos practicados para depositar las piedras del olivar que entorpecían las labores agrícolas, como se ha podido comprobar en el sector de excavación y en diferentes zonas del cerro.

\section{Contextualización histórica de la zona}

Como ya se ha apuntado, el lugar donde se localiza el asentamiento es una zona amesetada y sobrealzada, próxima al Cortijo de Tiena la Alta, cortijada que se ha mantenido sin grandes alteraciones hasta nuestros días a diferencia de la cortijada de Tiena la Baja, que hoy día es el núcleo primigenio del municipio de Tiena. Gracias a su situación, debió de gozar de las ventajas de una zona bien comunicada por vía terrestre y con abundantes recursos naturales: bosque de encinares principalmente (hoy desaparecido), tierras aptas para el cultivo, fuentes de agua. En este sentido, $\mathbf{M}^{\mathbf{a}}$. A. Marín señala en esta zona la existencia de vías secundarias que, a través de los pasos naturales, ponían en contacto el Municipium Florentinum Iliberritanum y el Municipium Ilurconensis, ambos en la Vega de Granada, con Anticaria (Antequera) y Castulo (Linares) (Marín Díaz, Mª A. 1988, 116). La proximidad y fácil acceso a dichas vías de comunicación era un factor determinante para este tipo de asentamientos rurales.

Por su ubicación geográfica la zona donde se enclava debió de depender administrativamente del territorio del municipio ilurconense, cuyos restos se localizan en el Cerro de los Infantes (término municipal de Pinos Puente). La situación administrativa de la villa del Cortijo de Tiena la Alta, subordinada al control fiscal del municipium durante el Alto Imperio, debió de ser común a otra serie de enclaves de similares características y muy próximos a la mencionada villa. Entre éstos se conoce la villa romana del Haza de los $\operatorname{Prados}^{4}$ en Olivares (Toro, I., 1987, 26), el yacimiento de época romana del Cortijo de Baeza (Tiena) ${ }^{5}$, el Cortijo de Búcor

\footnotetext{
${ }^{4}$ Excavación de urgencia realizada en el verano de 1987 por María del Pino de la Torre en la que se documentó un pavimento de opus espicatum y varios muros con restos de haber estado estucados. Por el material cerámico recuperado, terra sigillata hispánica y clara, lo situaron cronológicamente entre los siglos I al IV d.C. Y dadas las características constructivas de la obra posiblemente corresponda a la pars rustica de la villa. La ausencia de la publicación de la memoria de excavación no ha hecho posible la recopilación de más datos acerca de éste asentamiento, uno de los pocos excavados con metodología arqueológica en la zona de estudio.

${ }^{5}$ De este yacimiento sólo se conoce la procedencia de una inscripción funeraria hallada en 1974, que ha sido fechada por sus investigadores en la segunda mitad del s. I a.C. (Pastor, M. y Mendoza, A. 1987, 209). Respecto a esta propuesta cronológica, J. González considera la existencia de algunas villae romanas por estos lugares y la consiguiente romanización de la zona en época relativamente temprana (González, J. 1976, 393).
} 
(Pellicer, M., 1962-64, 311) y los restos de edificación y musivarios documentados por Gómez-Moreno en una de las márgenes del río Cubillas (Gómez-Moreno 1949, 373). Además se tiene constancia de la existencia de un asentamiento, de época romana, también de carácter rural, coronando un elevado cerrete no muy lejos de la villa del Cortijo de Tiena la Alta ${ }^{6}$.

\section{Secuencia estratigráfica}

Tras el estudio, tanto de los distintos materiales arqueológicos recuperados (Fig. 4) como de las relaciones que se establecen en el registro arqueológico entre las distintas unidades estratigráficas, se ha procedido a la estructuración de la secuencia estratigráfica de la zona excavada. Zona que corresponde a una estancia rectangular, pavimentada con un suelo de opus tesselatum. A esta habitación se accede desde el atrio a través de una puerta, de la que sólo se conserva el umbral. A ambos lados de esta habitación se han documentado muy parcialmente otras dos estancias. Con toda posibilidad, estas estructuras forman parte de la pars urbana de la villa (Fig. 3 y Lám. 1).

En primer lugar, en el sector de excavación practicado se registra la aparición del substrato geológico a muy escasa profundidad, en una zona del cerro donde el llano amesetado del mismo se acaba y empieza la pendiente de la ladera. Este substrato geológico se caracteriza por ser una formación de conglomerados no cementados, de pequeño diámetro y envueltos por una matriz de textura arenosa.

\section{Fase I. Romano Altoimperial (s. II-III d.C.)}

Esta fase altoimperial engloba el momento de construcción y habitación del edificio excavado. Por lo que respecta al levantamiento del edificio se hizo necesario el uso de un sistema de acondicionamiento y aterrazamiento del terreno donde iba a ser ubicado. Aunque más que un sistema de aterrazamiento propiamente dicho, con muros de contención, se debería hablar de un enrasamiento o nivelación del terreno de escasa entidad, lo suficiente para aminorar las irregularidades del substrato geológico y conseguir una superficie horizontal sobre la cual se pudiera realizar la construcción (UEC. 41).

\footnotetext{
${ }^{6}$ Este dato ha sido corroborado por la visita realizada al propio yacimiento. Además de su localización tuvimos constancia oral, por la gente del lugar, que hace unos años se descubrió allí mismo un mosaico al practicar una fosa para el vertido de basura. Sobre el terreno sólo pudimos certificar la existencia de dicha fosa, colmatada de piedras y desechos, así como la gran cantidad de material cerámico y restos constructivos repartidos por todo el cerro.
} 
En dicho substrato se realizaron las fosas de cimentación de los muros que conformaban las habitaciones. Los muros de la habitación I, donde se halló el mosaico, están hechos a base de sillares bien escuadrados (muro sur -estructura 8- ${ }^{7}$, y de un mampuesto de cantos de piedra de mediano y gran tamaño cementados por una matriz de cal (muros este y oeste -estructuras 10 y 11 respectivamente-). Formando parte de la composición de esta argamasa se han hallado algunos fragmentos de cerámica romana, principalmente sigillata hispánica, que se ha tenido en cuenta a la hora de hacer la valoración cronológica de esta fase. El muro de cierre de esta habitación hacia el Norte no se ha localizado, posiblemente haya desaparecido debido a que en esta parte se encuentra el inicio de la pendiente del cerro lo que ha acelerado el proceso de erosión del mismo.

Los cuatro muros debieron de configurar una estancia rectangular, de aproximadamente $100 \mathrm{~m}^{2}$. Todos los muros se encuentran arrasados casi hasta nivel de cimentación, a excepción del muro de sillares que conserva parte del zócalo. Pero en ningún caso se conservan restos de pintura mural in situ, tan sólo vestigios de la capa de preparado que albergaba la pintura estucada.

En el muro sur se abre una puerta de $3 \mathrm{~m}$. de luz. El umbral (estructura 9) está construido con 2 losas de mármol gris de 1'5 m. de longitud cada una, flanqueadas por dos sillares. Ambas presentan un buen acabado. Destacan dos perforaciones circulares en los extremos de cada una, y que hacían la función de gozne de la puerta. En el interior de ambos goznes se encontraron sendas aplicaciones de hierro o ejes verticales de $8 \mathrm{cms}$. donde encajaban dos ejes sobre los que debieron girar ambas hojas de la puerta ${ }^{8}$.

El suelo de la habitación I se encontraba pavimentada con un mosaico (estructura 5). Para la instalación del opus tesselatum se dispusieron cuatro capas de preparado. La primera capa estaba elaborada exclusivamente por chinarro de tamaño fino (UEC. 41) para nivelar las irregularidades del terreno, que podía variar, según la zona, entre $10 \mathrm{y}$ $25 \mathrm{cms}$. de espesor. Sobre esta capa se disponía otra de cal, cerámica machacada y arena de río bien tamizada, de color rosáceo de $1 \mathrm{~cm}$. de espesor (UEC. 16). La siguiente capa estaba compuesta de cal y arena fina de color grisáceo de $0 ` 5 \mathrm{cms}$. (UEC. 15), sobre la que descansaba otra, también de cal y arena, a la que se añadió cerámica machada de color marrón de $1 \mathrm{~cm}$. de grosor (UEC. 17). Y encima de esta se encontraban las teselas

\footnotetext{
${ }^{7}$ Algunos de los sillares que componen este muro parecen ser claramente reutilizados de otras construcciones anteriores, también de época romana.

${ }^{8}$ Destaca el hecho de que los umbrales de la mayoría de las estancias pavimentadas con mosaico de la villa de Daragoleja presentan un sistema de cerramiento de características muy similares a las de la habitación excavada.
} 
del mosaico, trabadas con un fino mortero de cal y fina arena de color blanquecino. Formando parte de las capas de mortero se pudieron recuperar algunos fragmentos de cerámica común romana y sigillata hispánica amortizados en la obra, no obstante poco representativos, que podrían dar una cronología del s. II d.C. para el momento de construcción, aunque con grandes reservas por nuestra parte.

El alzado de los muros de la habitación I a partir del zócalo de sillares o de mampuesto de piedras debió de estar hecho a base de tapial, como se ha podido comprobar en los escasos derrumbes excavados (principalmente en el muro Este), recubiertos con un mortero de cal y arena que albergaría la pintura mural en su capa superficial.

Dadas las grandes dimensiones de la estancia se hace difícil pensar en la forma que resolvía el sistema de cubrición. En este sentido se han excavado dos agujeros de poste (UEC. 42 y 43), dispuestos a 1'80 m. de distancia delante de los dos sillares que flanquean la entrada. El fondo de dichos agujeros, de $0^{\prime} 15 \mathrm{~m}$. de diámetro, conservaba una piedra más o menos plana. Sin duda debieron formar parte del sistema de sustentación del armazón de la cubierta.

A ambos lados de la habitación I se han descubierto otras dos habitaciones: habitación II y III. Se desconocen sus dimensiones al no haber sido excavadas en extensión, tan sólo una estrecha franja que corre paralela a los muros medianeros que comparten con la habitación I. En la habitación III, se ha documentado un pavimento de opus caementicium, el cual se encuentra bastante erosionado y perdido por completo allí donde empieza la pendiente del cerro (estructura 7).

Anteponiéndose a lo largo de toda la extensión de la línea de fachada sur de la habitación I encontramos un pavimento de opus caementicium (estructura 6) adosado al muro y a las losas de mármol de la entrada. Es por tanto desde esta zona abierta, posiblemente porticada, desde donde se accedía tanto a dicha habitación, como también al resto de las estancias que se hallaban abiertas al patio central de la pars urbana. El suelo, hecho a base de fragmentos de cerámica trabados con cal y arena y algunas piedras, presenta una suave inclinación hacia el centro del patio, donde debía de existir una pequeña alberca o cisterna.

Estas estructuras pudieron estar en uso hasta un momento no más allá del s. IV d.C, momento a partir del cual se produce la ruina de las mismas.

\section{Fase II. Romano Bajoimperial (s. IV d.C)}

A esta fase corresponde el abandono y destrucción de la parte de la villa excavada. A partir de este momento el registro va a sufrir una serie de procesos postdeposicionales, principalmente erosivos, y recientemente los derivados de las labores agríco- 
las. Éstos han incidido negativamente de manera directa en la conservación y preservación de los restos. A través de dichos procesos, en unos casos, se han eliminado casi por completo los niveles arqueológicos correspondientes a esta fase, dispersando su material por todo el cerro, y en otros, los niveles sedimentarios se han visto alterados en su estructura interna por remociones antrópicas que además han acabado con jnás de las dos terceras partes del mosaico.

Como ya se ha apuntado, esta fase viene definida por los derrumbes que se han documentado en las distintas zonas excavadas, tanto en la habitación I como en la habitación III y en el atrio. Hay que tener en cuenta que el nivel estratigráfico que corresponde con los niveles de derrumbe no son homogéneos a toda la superficie excavada, ya que se encuentran fuertemente alterados tanto por el arado como por las fosas realizadas para plantar olivos o esconder piedras, además de la limpieza del mosaico previa a esta intervención.

En la habitación I estos niveles están representados por los correspondientes al derrumbe de las estructuras de tapial y piedras procedente del muro este (UEN 11 y UEN 14 respectivamente). Es una tierra de color naranja amarillento, fácil de distinguir del resto de las tierras, y que procede de la degradación del tapial que configuraba el alzado de los muros de la habitación. Formando parte de dicho derrumbe se encuentran derrumbes del estucado de la pared, los cuales formaban parte del sistema decorativo.

A esta fase de derrumbe se puede adscribir un nivel de incendio (UEN. 13) en la habitación III, que se extiende a lo largo de todo el pavimento excavado. La escasa potencia estratigráfica que presenta esta estancia no ha permitido la conservación de niveles correspondientes al proceso de derrumbe. Este nivel de incendio ha servido para datar la destrucción y/o abandono del edificio en torno al s. IV d.C. Para ello se ha tenido en cuenta la cronología relativa que nos aporta el conjunto cerámico que formaba parte de dicho nivel, compuesto principalmente por sigillata hispánica tardía, sigillata africana A y C, cerámica común y de cocina romana, etc. En este sentido, el fragmento más reciente no sobrepasa esta fecha ${ }^{9}$.

En el atrio también se ha documentado el mismo proceso de derrumbe que en la habitación I, tanto de tapial (UEN. 6) como de estuco (UEN. 9), presentando además, un derrumbe de tégulas (UEN. 10) mejor conservado.

\footnotetext{
${ }^{9}$ Fragmento de Sigillata Africana C forma Lamboglia 40 (primera mitad del S. III al S. IV d.C.).
} 


\section{Fase III}

En esta fase estratigráfica se ha englobado un nivel de tierra (UIEN. 7) que se ha interpretado como un nivel de cultivo antiguo que aún conservaba, aunque muy desdibujados, los surcos dejados por el arado. Se ha datado en época moderna, aunque el tipo de cerámica vidriada no es tan significativa para concretar más.

\section{Fase IV}

A esta fase corresponden la tierra de cultivo actual y las distintas fosas practicadas en el registro arqueológico en los últimos años.

\section{Análisis estilístico del mosaico}

Los restos de mosaico que se han puesto al descubierto en la villa romana del Cortijo de Tiena la Alta se encontraban en un estado de conservación bastante bueno si tenemos en cuenta las condiciones postdeposicionales tan adversas a las que nos hemos referido anteriormente. Estas mismas circunstancias (escasa potencia estratigráfica para amortiguar cualquier agresión, los trabajos del arado de la zona, etc.) son las causantes de la pérdida de más de dos tercios del pavimento musivario, a lo que además hay que añadir la acción de la cal de los restos constructivos que forman parte del registro (estucos y morteros), causante de fuertes concreciones en algunas zonas del mosaico.

El mosaico está hecho con pequeñas teselas de $1 \times 1 \mathrm{~cm}$., aunque puede variar el tamaño en algunos casos sensiblemente. Están bien recortadas y sin apenas separación entre ellas, por lo que el fraguado ofrece un buen acabado. Las teselas son de mármol (carbonato cálcico) de color negro y blanco. Los análisis efectuados a las teselas ${ }^{10}$ (difracción de R.X.) demuestran que la composición de éstas es de CO Ca3 aproximadamente al $100 \%$ de pureza. La diferencia en el color, teselas blancas o negras, es debido a un pigmento de origen orgánico, cuya identificación exacta no ha sido posible.

El paso del tiempo ha provocado leves rehundimientos puntuales del preparado y por consiguiente de la superficie del mosaico. Ocupa una superficie total de $20^{\prime} 84$ $\mathrm{m}^{2}$. El tema principal presenta una decoración conseguida a base de motivos geométricos: círculos secantes entre sí de $30 \mathrm{cms}$. de diámetro, también llamado cuatripétalos o cuadrilobulados, debido a que en las intersecciones se dibujan unas hojas que a su vez forman una roseta de 4 hojas en color negro sobre fondo blanco y

\footnotetext{
${ }^{10}$ Análisis realizados por Gabriel Delgado del Departamento de Edafología y Química Agrícola de la Facultad de Farmacia, Universidad de Granada.
} 
cuadrados curvilíneos, o de lados cóncavos de color blanco, que albergaban en su interior un cuadrado en color negro y a su vez un cuadrado dentado en blanco timbrado con una tesela negra en su centro (Lám. 2).

El tema central se halla enmarcado por una orla en la que se representa una cenefa de hojas de agua de las que parte un tallo en espiral que va enlazando unas con otras a modo de roleos. Estas hojas de agua van alternando, cambiando su orientación arriba y abajo (Lám. 2). Dichos roleos parten de un motivo floral central que se encuentra justo en medio de la entrada de la habitación, delante del umbral (Fig. 5).

Este motivo de los círculos secantes se documenta en Hispania a partir el s. II d.C. de manera esporádica, difundiéndose rápidamente en los siglos III y IV. Es uno de los más comunes en la musivaria romana, apareciendo tanto en la parte occidental como en la oriental del Imperio (Fernández Galiano, D., 1984, 221). La variante de la temática de círculos intersecantes más sencilla, (y probablemente también la más antigua), es la realizada en blanco y negro, en la cual las intersecciones que forman cuadripétalos están hechas con teselas de color negro, sobre fondo blanco (Járrega Domínguez, R. 1993, 279). Dicha variante es la documentada en el mosaico que nos ocupa.

Preferentemente, este tema decorativo, se colocaba en las zonas de ambulacro y como campo central, al que se le podía insertar en ocasiones un emblema figurado y siempre enmarcado. Excepcionalmente este motivo era colocado como orla-tema de enmarque en hilera de círculos (Mérida o Balazote) o como panel adyacente compartiendo su protagonismo con otro tapiz geométrico de diferente temática (Muñoz Jofré, L. 1996,143). En este caso los círculos secantes ocupan de forma monográfica el campo central, y aunque no se tienen indicios, no se descarta la posibilidad de que hubiera existido un emblema figurativo inserto en esta composición.

Los paralelos más directos que se conocen en la provincia de Granada son los de la villa de Daragoleja, los de la villa de C/ Primavera, C/ San Juan de los Reyes y la villa que cita Gómez-Moreno en Pinos Puente, en las proximidades del Río Cubillas. En todos ellos el tema decorativo de los círculos secantes está presente en mayor o menor medida, pudiendo formar parte del campo central.

Éste es el caso del Mosaico de los Rosetones y del corredor de la villa de Daragoleja $^{11}$ (Gómez-Moreno, M. 1949, 381 ss. y Blázquez, J. M. 1982, 41 ss.) y del mosaico de la villa del Río Cubillas, perteneciente posiblemente al impluvium. En

${ }^{11}$ Excavada en 1870 y datada en los siglos IV-V d.C. por M. Gómez-Moreno. Se puso al descubierto un largo corredor de unos $20 \mathrm{~m}$. de longitud, de opus tesselatum, desde el que se accedía a varias estancias, algunas de las cuales también estaban pavimentadas con mosaicos policromos, entre las que se encontraba el triclinium, hoy perdidos. Los motivos decorativos principales eran enlaces, rosetas, triángulos enfilados, trenzas, círculos, etc. 
otros casos, este motivo aparece ocupando una posición secundaria, como en el triclinium y habitación del Mosaico con Frutos y Ave de la villa de Daragoleja. Y como caso más excepcional, formando parte de la composición ornamental de la orla del mosaico. Hecho constatado en los escasos restos documentados en la C/ San Juan de los Reyes de Granada, donde el motivo forma una hilera de círculos secantes.

En Hispania tuvo una gran aceptación durante la segunda mitad del s. III y principios del s. IV d.C. como lo demuestran los hallazgos arqueológicos inventariados, momento en el que alcanza su cénit estadístico, junto al renacer económico de los grandes possessores de villas, reconociendo su posterior declive y desaparición durante los siglos V y VI. Además se ha documentado en 36 pavimentos procedentes en su mayoría de las grandes villas del Bajo Imperio (Muñoz Jofré, L. 1996,137-141).

Entre los ejemplos documentados en la Bética destacamos los de Córdoba, como el mosaico de la Casa de Mitra en Cabra, el mosaico de Eros y Psique de Córdoba (Blázquez, J. M., 1981, 35), o los mosaicos de la Plaza de Corredera (García y Bellido, 1965, 185) y Mosaico de Pegaso de la Calle Cruz Conde, 20, en los cuales el tema decorativo mayoritariamente dominante son los círculos intersecantes junto a algunas cenefas con roleos de ramitas ondulantes como el de la Avda. del Generalísimo.

En la provincia de Sevilla son también numerosos los casos en los que al menos uno de los temas decorativos predominantes son los círculos secantes. Como el mosaico con Triunfo de Dioniso ¿Baco? de Écija (Sevilla) (Blázquez, J. M., J. M. 1982, 13), el mosaico del corredor con alfombra del Triunfo de Baco de Itálica en Casa de la Condesa de Lebrija (Sevilla), el mosaico de Galatea de Itálica (Sevilla), los mosaicos de Carreras de Carros (A) y con Cuadriga (B) enmarcados por una orla de círculos secantes. Actualmente se encuentran en el Museo Arqueológico Provincial de Sevilla aunque fueron hallados en el Cortijo de Paterna (Blázquez, J. M., 1982, 19). En la provincia de Málaga destacamos el mosaico de San Pedro de Alcántara y el de la villa romana de las Torres de Estepona (Blázquez, J. M. 1981,93) y en la provincia de Jaén los mosaicos de la villa de Bruñel (Muñoz Jofré, L. 1996).

Por lo que respecta a la orla, el tema principal es un motivo floral esquematizado, presidiendo la entrada a la habitación I, del que surgen dos tallos, en cuya espiral van enlazando una cenefa de hojas de agua. Este tipo de motivos decorativos son característicos de las orlas y cenefas de enmarque de los temas principales en las composiciones musivarias, por lo que muy variados son los ejemplos donde aparecen y muy diversas las combinaciones que adquieren entre los distintos componentes. Este hecho hace casi improbable hallar dos orlas gemelas. Como ejemplo más próximo a la villa del Cortijo de-Tiena y con el que se puede establecer una correspondencia comparativa más directa es el mosaico II de la villa de la C/ Primavera (Fresneda et alii 1991, 150). Y aunque no 
es exactamente igual existe entre ambos un gran paralelismo no sólo estilístico sino también técnico, sobretodo en la forma en la que está elaborada la banda de espirales entrelazadas, también en negro sobre fondo blanco. La diferencia más acusada es la ausencia de un elemento central del que broten los roleos o tallos en espiral.

Fuera de la provincia de Granada se conocen bastantes muestras de orlas de este tipo, aunque sólo destacaremos la cenefa con hojas de hiedra en negro sobre fondo en blanco del mosaico de Eros y Psique de la Avda. de Córdoba o la cenefa con roleos que enmarca el tema central del Mosaico de Pegaso de la C/ Cruz Conde también de Córdoba.

\section{Tratamiento de conservación, limpieza, extracción y embalaje del mosaico}

Estado de conservación. Una vez excavado el mosaico en toda su extensión se pudo comprobar el estado de conservación en el que se encontraba. En primer lugar hay que destacar las faltas de considerable tamaño, producidas por las labores agrícolas de la zona, anteriormente mencionadas, tanto de material pétreo como de mortero. También se apreciaron disgregaciones en zonas de fractura de la superficie y una gran inestabilidad en los bordes de las lagunas, con desprendimientos muy acusados de teselas. En algunas zonas, pequeñas porciones del mosaico se encuentran invertidas y revueltas debido principalmente a la acción del arado. Además hay algunos rehundimientos, producidos por fuertes golpes al caer sobre la superficie los restos de muros y de cubierta de la habitación y abombamientos de la superficie causados por tensiones de tipo mecánico debidas a las raíces de olivo. Este problema es particularmente acusado en la zona del umbral de la puerta. Pero el problema que más atención ha necesitado ha sido la existencia de concreciones carbonatadas, que cubrían gran parte de la zona oeste del mosaico.

Consolidación, limpieza y extracción del mosaico. Para este trabajo se ha contado con un equipo de restauradores, auxiliados por el equipo de arqueólogos a lo largo de todo el proceso. Una vez excavado el mosaico en toda su extensión, en primer lugar, se llevó a cabo una limpieza general empleando un cepillo de cerdas de nylon. A continuación se realizó saneamiento y limpieza de los bordes y límites del mosaico, inclusive los de las lagunas, para la consolidación de aquellas partes que se encontraban dañadas o desmontadas por el paso del tiempo y del arado. Dicha consolidación consistió en la fijación con un acetato de polivinilo (APV) diluido al $50 \%$ en agua desmineralizada de aquellas teselas que se encontraban sueltas o a punto de soltarse. Con esta operación, queda asegurado el que las teselas no se suelten al tener que manipular posteriormente los bordes para el arranque. Igualmente, al inyectar el adhesivo, éste penetra hasta el mortero, consolidándolo a la vez. A continuación se consolidaron todos los bordes de las lagunas y faltas con una tabicación de escayola. 
Tras el proceso de consolidación se practicó una limpieza superficial de grado medio con agua desmineralizada, mezclada con alcohol (etanol) en la proporción 1:1 y cepillos de raíces. A esta mezcla, se le ha añadido unas gotas de agente tensoactivo (Teepol) para que ayude a limpiar, y una disolución al 3\% aproximadamente de Fenol, que hace de fungicida. El proceso consiste en humedecer la superficie con esponja impregnada con esta solución, seguidamente se cepilla fuertemente, enjuagando y secando posteriormente el mosaico con bayetas absorbentes. Esta operación se realizó repetidas ocasiones, En la mitad oeste del mosaico la presencia de fuertes concreciones calcáreas retrasaron la limpieza. Para su eliminación se hizo necesaria una limpieza de tipo mecánico-manual, con ayuda de los bisturíes, lápices de fibra de vidrio y lana de acero inoxidable. Como testimonio de las fuertes concreciones anteriormente existentes se dejaron dos pequeños testigos.

Una vez limpio y desalado, se documentó tanto fotográfica como planimétricamente a E. 1:1 y 1:20 (Lám. 1 y Fig. 3). Posteriormente se engasaron los bordes y los límites de las lagunas. A continuación se encoló el mosaico para su engasado con un adhesivo (acetato de polivinilo) al 100\% muy reversible. El engasado es una operación protectora del mosaico, encaminada a poder manipular a posteriori el mosaico, sin que sufra desperfectos ni pérdidas importantes durante el arranque (Lám. 3). Tras diseñar los cortes sobre el dibujo se realizó la segmentación del mosaico siguiendo el motivo decorativo. Y con ayuda de unas "espadas" se procedió a la extracción de los distintos fragmentos de mosaico, previamente maceados y una vez numerados los trozos. El proceso de extracción es la parte más delicada y de ella depende su conservación posterior. Cuando se tiene cada fragmento separado por completo del resto del mosaico, se deposita sobre un tablero de aglomerado embalándolos a modo de "sandwich", donde se rotularon las siglas identificativas para su depósito en el Museo Arqueológico Provincial de Granada.

\section{Conclusiones}

Como se ha indicado anteriormente, las gestiones realizadas en el 1995 dieron como resultado el comienzo de los trabajos arqueológicos en octubre de 1997. Una vez finalizados los trabajos de urgencia, los resultados arqueológicos que se han ido obteniendo han rebasado cualquiera de las expectativas que se tenían presentes antes de iniciar la excavaciones de los restos. Los escasos $3 \mathrm{~m}^{2}$. de mosaico que se descubrieron en un primer momento han resultado tener en realidad una extensión de más de $20 \mathrm{~m}^{2}$. pudiéndose enmarcar el hallazgo dentro del contexto arqueológico al que pertenece. Como se ha adelantado anteriormente, el tema del mosaico fue bastante corriente en época romana, sobretodo en el s. III d.C., de gran sencillez y concepción 
simétrica en su composición. Motivo que fue empleado en la mayoría de los restos musivarios conocidos en Granada y en el resto de la Bética, sin mencionar el resto de Hispania o del Imperio. La repetición no sólo de los motivos decorativos ${ }^{12}$ sino también las analogías que se pueden establecer en sus aspectos técnicos pueden hacernos pensar en la existencia de un taller de artesanos musivarios que trabajara en el ámbito de los municipios iliberritano e ilurconense.

En cuanto al contexto arquitectónico donde apareció dicho mosaico, éste corresponde a una habitación, de otras tantas que debieron de conformar la parte de la villa romana perteneciente a la pars urbana. Esta zona era el área dedicada a vivienda. Su estructuración en muchos casos era bastante similar a la de las casas urbanas de las ciudades (domus). Normalmente, las distintas estancias estaban estructuradas en torno a un espacio abierto que podía ser un patio (atrium). La estancia que se ha puesto al descubierto, dadas las características que presenta y que se han comentado más atrás, debió de ser una de las habitaciones principales de la pars urbana, posiblemente el oecus. Para argumentar esta idea no sólo se ha tenido en cuenta la pavimentación de la misma, conseguida a base de un pavimento musivario de excelente factura, sino también por el material de construcción empleado, principalmente sillares bien escuadrados en algunas partes de sus muros, junto con los restos de pintura mural. Pero tal vez, lo que más ha sorprendido, además de las características constructivas señaladas anteriormente, son las dimensiones, envergadura y localización de la construcción. Presenta 10 m. en su línea de fachada sur por más de $9 \mathrm{~m}$. en sus flancos E y O. Además se puede tomar como referencia de ello el umbral de la puerta de acceso a la habitación, que presenta $3 \mathrm{~m}$. de longitud. Y aunque se desconoce la estructuración arquitectónica general de la villa en toda su planta, las dos habitaciones que se han documentado tímidamente a los lados E y O junto con el pavimento de opus caementicium que se antepone a toda la fachada por el $\mathrm{S}$ de la habitación, probable atrio porticado de la villa y a donde se abre la estancia, hacen pensar en la posición central, presidiendo dicho atrio porticado, en torno al cual estarían ordenadas el resto de las habitaciones de la zona residencial.

Este hecho fue casi una norma durante el Bajo Imperio en la edificación de la vivienda noble, otorgando el lugar central y de mayores dimensiones a las habitaciones de más distinción social: triclinium y oecus, éste se situó en lugar destacado, presidiendo el peristilo. Hecho que quedará pendiente de confirmar hasta la excavación en extensión del resto de las habitaciones y espacios de la villa. Teniendo en cuenta esta premisa, el hallazgo de la estancia excavada no ha de entenderse aisladamente sino en relación con un contexto de mayor complejidad arquitectónica aún por descubrir.

\footnotetext{
12 En el caso de la orla de círculos secantes del mosaico de la C/ San Juan de los Reyes los círculos presentan el mismo diámetro.
} 
Por tanto, una vez estudiados tanto la secuencia estratigráfica como los restos cerámicos y establecidas las correlaciones estilísticas, formales y cronológicas con otras muestras musivarias de marcada analogía se ha podido establecer como momento inicial de la construcción del monumento el s. II d.C., teniendo en cuenta las debidas reservas, ya que estos criterios no ofrecen garantías sólidas para ello. Y para el momento de abandono y/o destrucción se ha tenido en cuenta el material cerámico procedente de los niveles de derrumbe de la habitación I y del nivel de incendio de la habitación III, no siendo posible prolongar mas allá del s. IV d.C. dicho acontecimiento.

Concluidos los trabajos arqueológicos se ha procedido a la cubrición de los restos emergentes, previamente tapados con geotextil, empleando para ello la misma tierra que anteriormente se extrajo durante el proceso de excavación. Esta actitud ha estado motivada por dos hechos principalmente: la protección de los restos de cualquier tipo de agresión y para eliminar los obstáculos que supone un corte en el terreno de estas características.

\section{BIBLIOGRAFÍA}

BLÁZQUEZ MARTÍNEZ, J.M. (1981): Mosaicos romanos de Córdoba, Jaén y Málaga. Madrid.

BLÁZQUEZ MARTÍNEZ, J.M. (1982):Corpus de Mosaicos de España. Fascículo IV. Mosaicos romanos de Sevilla, Granada, Cádiz y Murcia. Madrid. Instituto Español de Arqueología "Rodrigo-Cano" del Consejo Superior de Investigaciones Científicas.

BLÁZQUEZ MARTÍNEZ, J.M. (1993): Mosaicos romanos de España. Madrid.

FERNÁNDEZ GALIANO, D. (1984): “Complutum. Mosaicos II”. Excavaciones Arqueológicas en España. Pp. 217-222.

FRESNEDA, E.; TORO, I.; PEÑA, J.M.; GÓMEZ, R. y LÓPEZ, M. (1991): “Excavación de emergencia en la villa romana de la C/ Primavera (Granada)". Anuario Arqueológico de Andalucía III (1989). Sevilla. Pp. 149-156.

GARCÍA Y BELLIDO, A. (1965): “Los mosaicos romanos de la Plaza de la Corredera en Córdoba". Boletín de la Real Academia de la Historia CLVII, Cuad. II. Pp.183-189. GÓMEZ-MORENO, M. (1949): Misceláneas. Historia, Arte y Arqueología. Primera Serie: La Antigüedad. Madrid.

GONZÁLEZ FERNÁNDEZ, J. (1976): “Ilorci (Plinio N.H. 3,9)”. Habis 7. Madrid. Pp. 391 ss. 
JÁRREGA DOMÍNGUEZ, R. (1993): “El mosaico policromo con decoración geométrica de círculos intersecantes de la villa romana de Barrugat (Bitem, Tarragona)". AEspA 66. Pp. 275-284.

MARÍN DÍAZ, A. (1988): "Introducción al estudio de las vías romanas en la Provincia de Granada”. Vias romanas del SE. Coord. por A. González. Murcia. Pp. 113118.

MUÑOZ JOFRÉ, L. (1996): Elementos ornamentales de la Villa romana de Bruñel. Mosaicos y pintura mural. Vol. I y II. Tesis Doctoral. Universidad de Granada.

PASTOR MUÑOZ, M. y MENDOZA EGUARAS, A. (1987): La epigrafía latina de Granada y su Provincia. Granada

PELLICER CATALÁN, M. (1962-64): “Actividades de la delegación de zona de la provincia de Granada durante los años 1957-1962". N.A.H. VI.

TORO MOYANO, I. (1987): Memoria de Gestión de las Actividades Arqueológicas de la Provincia de Granada. 
Fig. 1: Mapa del término municipal de Moclín. Localización del yacimiento arqueológico
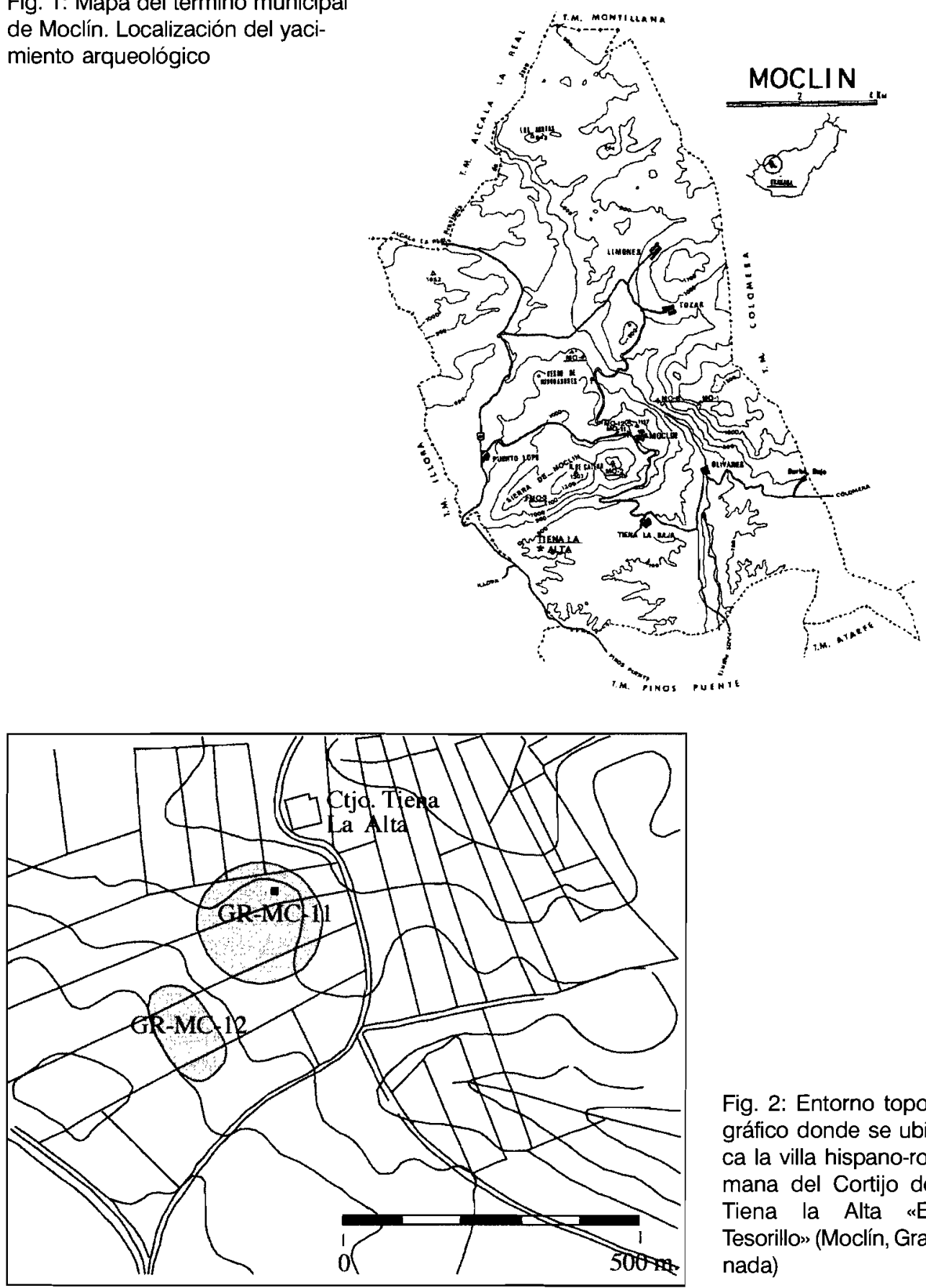

Fig. 2: Entorno topográfico donde se ubica la villa hispano-romana del Cortijo de Tiena la Alta «El Tesorillo» (Moclín, Granada) 


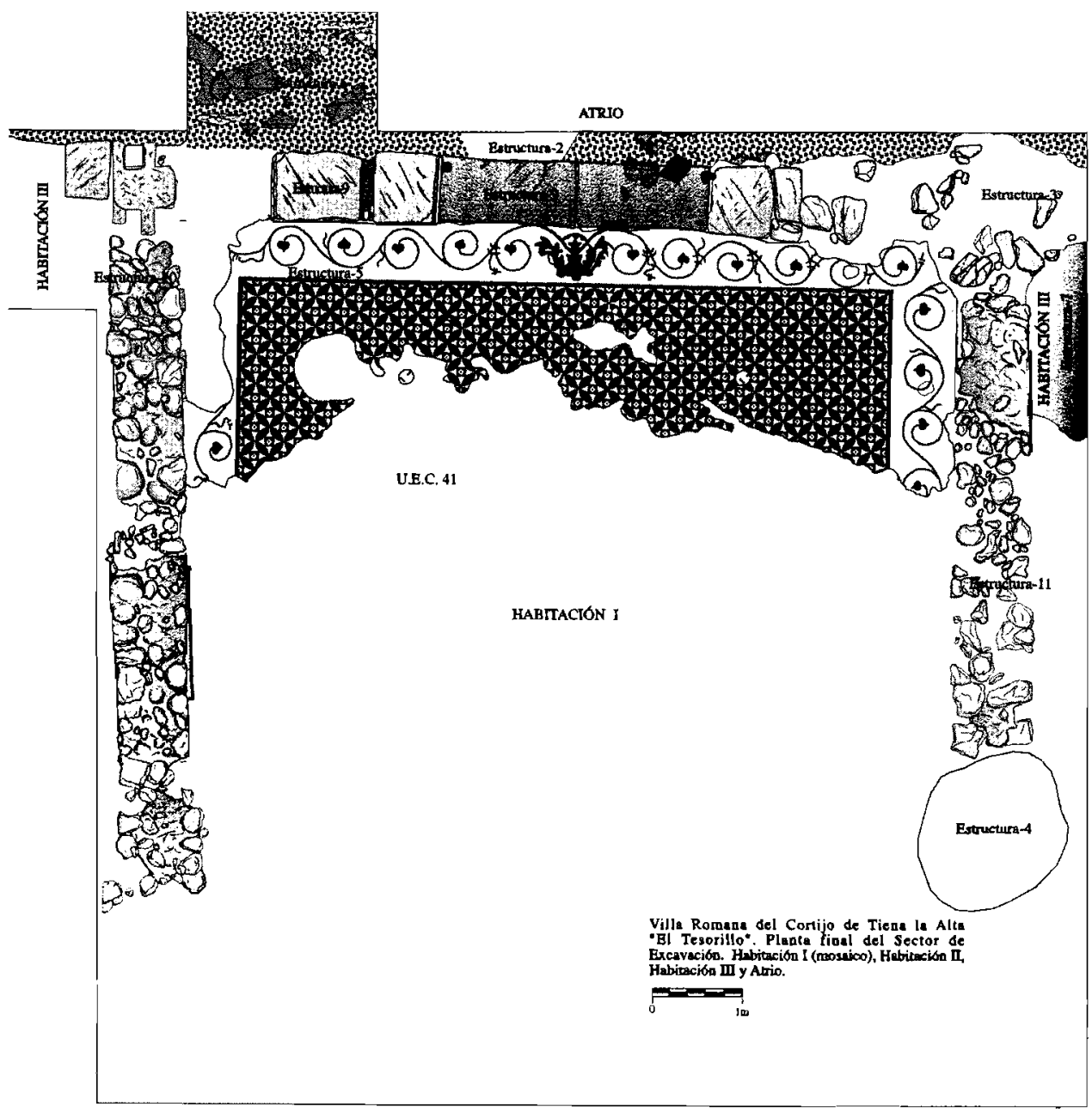

Fig. 3: Planta general del sector de excavación 

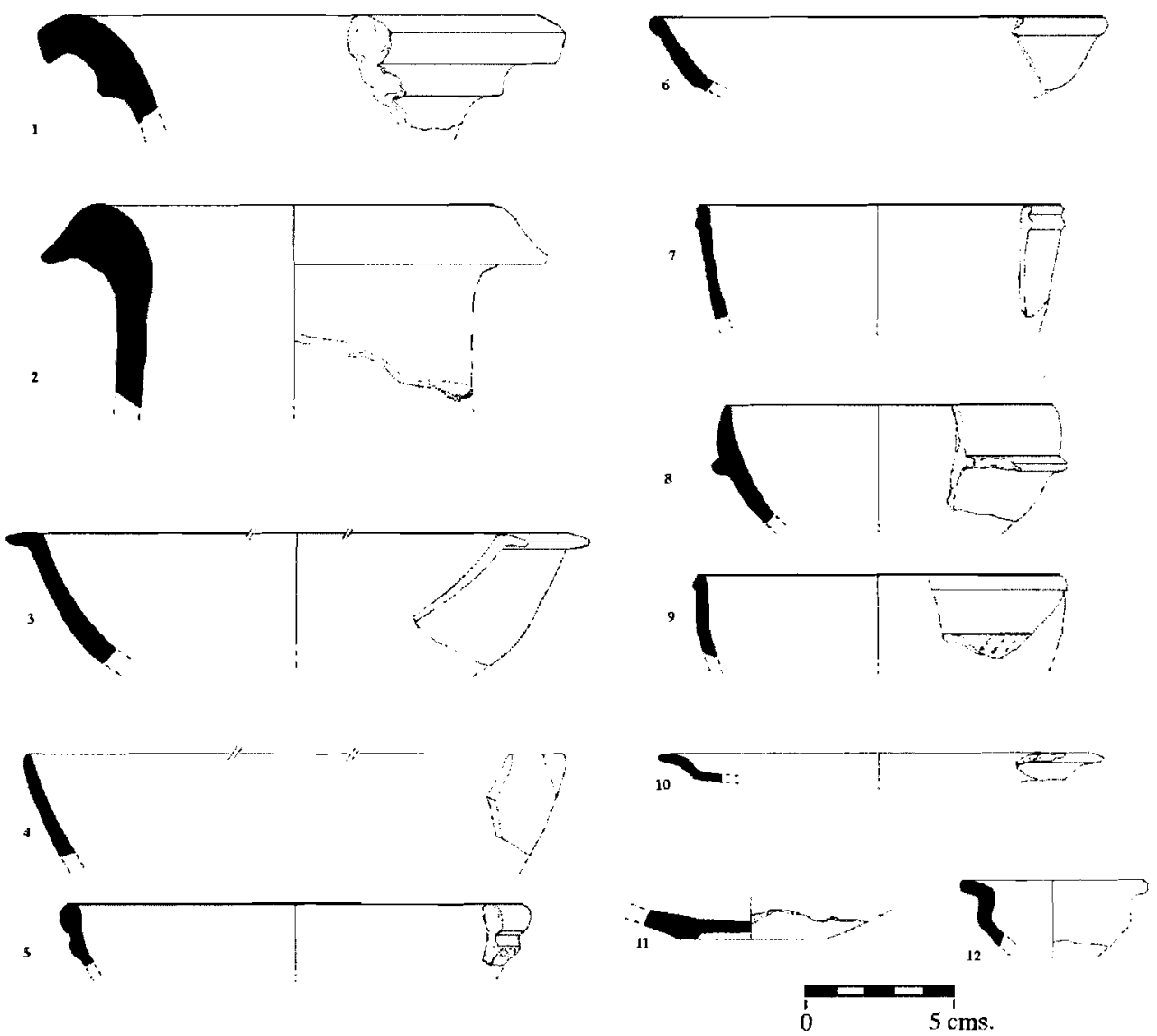

Fig. 4: Cerámica representativa de los distintos niveles. Ánforas 1 y 2; TSAC 3, 4 y 10; TSAA 5; TSHI 6, 7, 8, 9 y 11; común 12.

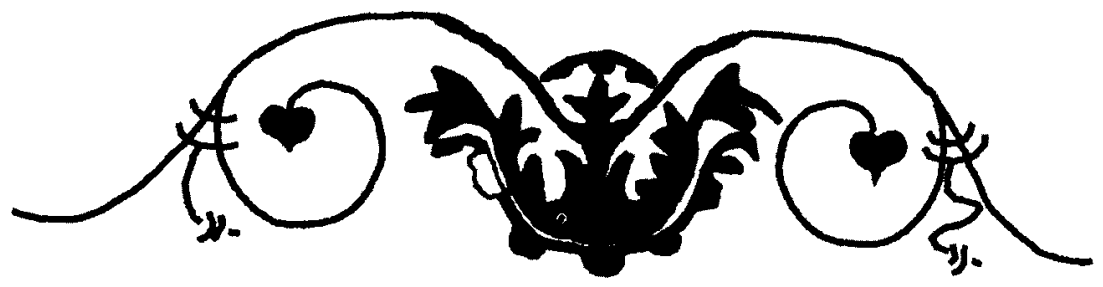

Fig. 5: Detalle del motivo central del mosaico que preside la entrada a la habitación 


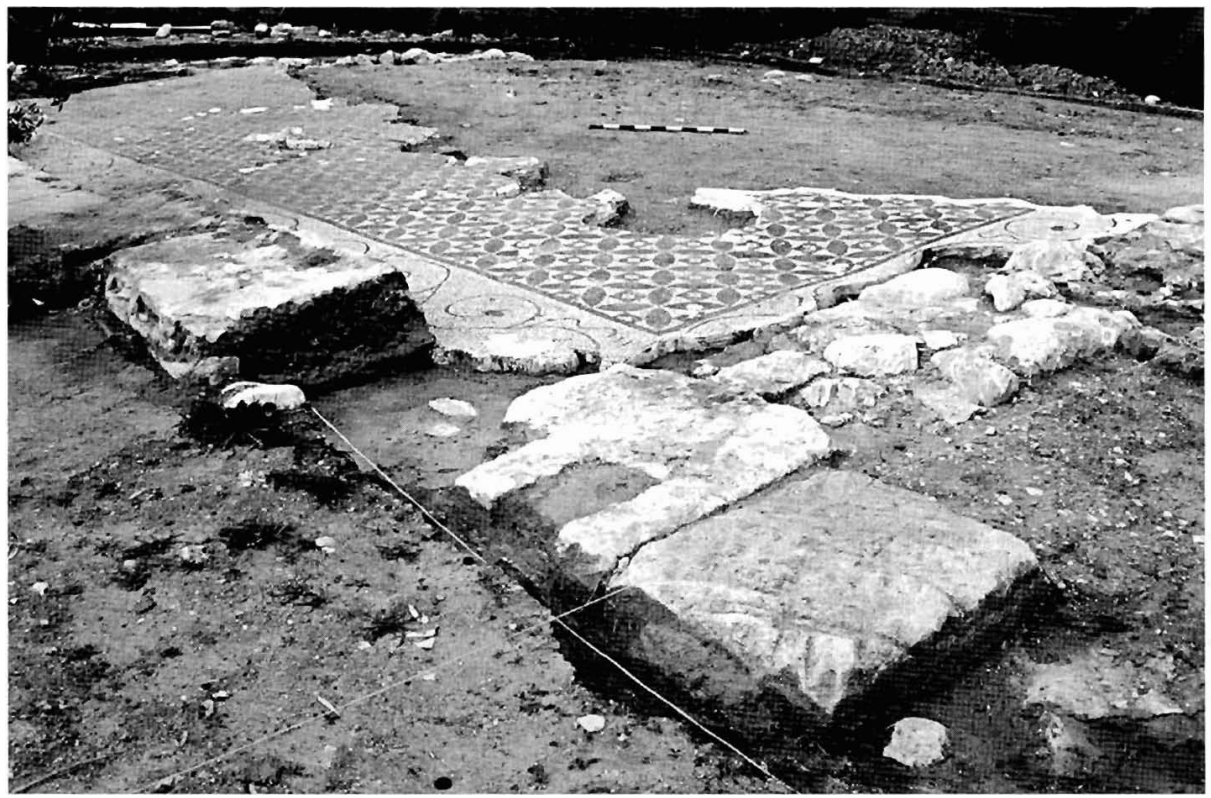

Lám. 1: Vista general de los restos excavados de la villa

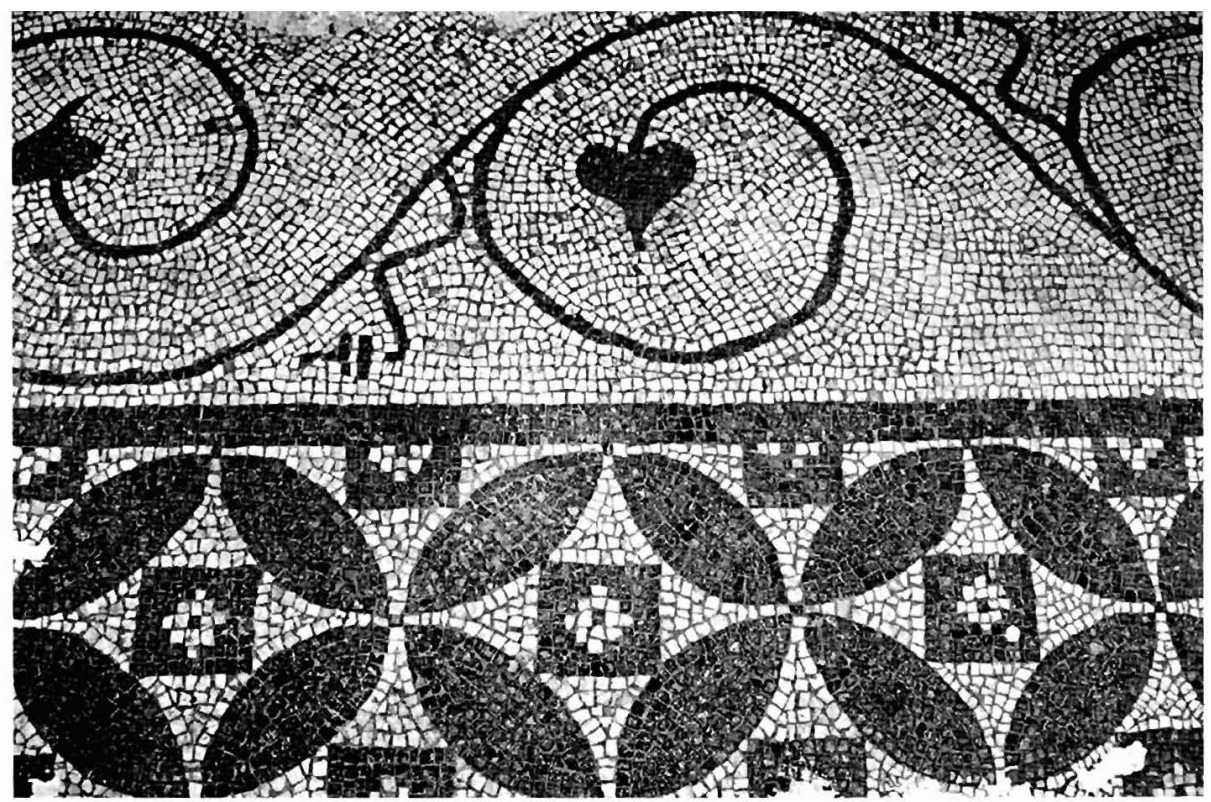

Lám. 2: Detalle del mosaico 


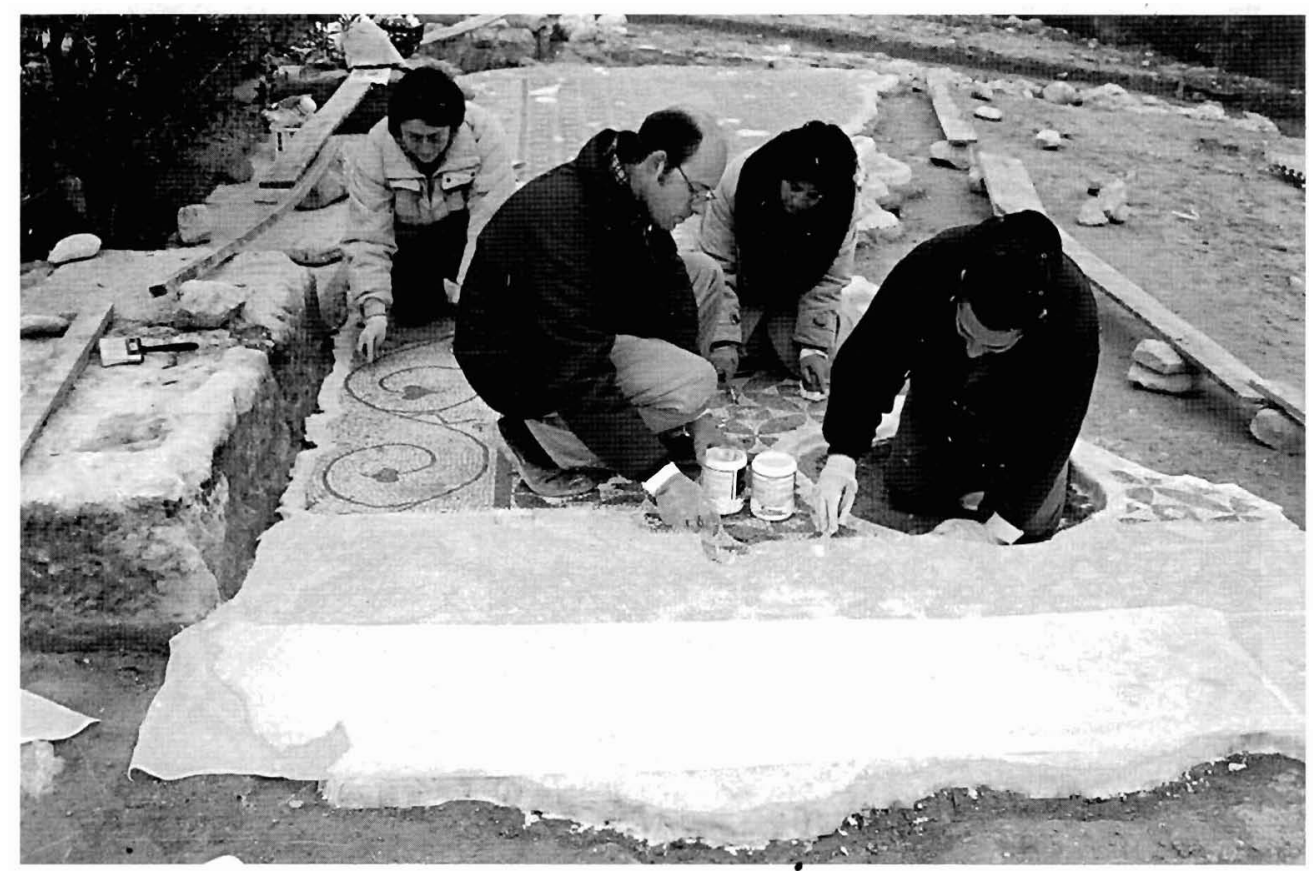

Lám. 3: Proceso de encolado y engasado, previo a la extracción del mosaico 